Chapter 29.

\title{
Legume Production in Florida: Snapbean, Lima Bean, Southernpea, Snowpea
}

\author{
S.M. Olson, E.H. Simonne, W.M. Stall, S.E. Webb, T.G. Taylor, S.A. Smith, A.J. Palmateer
}

\section{BOTANY}

\section{Nomenclature}

Family - Fabaceae (Leguminosae)

Snapbean - Phaseolus vulgaris

Lima bean - Phaseolus lunatus

Southernpea - Vigna unguiculata

Snowpea - Pisum sativum

\section{Origin}

Snapbean and lima bean are New World vegetables with Central America being the center of origin. Southernpea and snowpea originated in southeastern Africa and central Asia, respectively.

\section{Related Species}

All of the vegetable crops generally recognizable as peas and beans are included in the Fabaceae family. One root crop, jicama, is also a legume. Many species are of economic importance and the family is, after the Poaceae, the most important source of human food. It also provides field and forage plants, timber, fiber, dyes, gums, insecticides, flavorings and many other products. A number of ornamentals also are included in this family. The unique ability of legumes to fix atmospheric nitrogen is of great importance; however, it is not of much practical significance in the vegetable legumes because of the short crop cycle. Southernpea is more efficient in $\mathrm{N}$ fixation than snapbean.

\section{VARIETIES}

Selection of the variety to plant is one of the most important decisions the commercial vegetable grower must make each season. Each year seed companies and experiment stations release dozens of new varieties to compete with those already available. Growers should evaluate some new varieties each year on a trial basis to observe performance on their own farms. Plant only those that show real promise based on University of Florida, industry, or grower trials. A limited number of new varieties should be evaluated so that observations on plant performance, characteristics, and yields can be noted and recorded. It is relatively easy to establish a trial but very time-consuming to make all the observations necessary to make a decision on adoption of the new variety for large scale production. Some factors to consider before adopting a variety are:

Yield - The variety should have the potential to produce crops at least equivalent to those already grown. In recent years, the average yield of beans in Florida was about 180 30-lb bushels per acre. Harvested yield may be much less than potential yield because of market constraints.

Disease Resistance - The most economical and effective means of pest management is through the use of varieties that are resistant or tolerant to disease. Many bean varieties are tolerant of some strains of common bean mosaic. When all other factors are about equal, it would be prudent to select a variety with needed disease resistance or tolerance.

Horticultural Quality - Characteristics of the plant habit as related to climate and production practices and of the marketed plant product must be acceptable. Beans to be harvested mechanically must have sufficient pod wall fiber to protect the integrity of the product. Beans to be hand harvested are of lower fiber content and have higher eating quality.

Adaptability - Successful varieties must perform well under the range of environmental conditions usually encountered on the individual farm.

Market Acceptability - The harvested plant product must have characteristics desired by the packer, shipper, wholesaler, retailer, and consumer. Included among these qualities are pack out, size, shape, color, flavor, and nutritional quality. Consumers have identified dark-green pod color and small sieve size with high culinary quality.

Variety selection is a very dynamic process. Some varieties retain favor for many years, whereas others might be used only a few seasons if some special situation, such as plant disease or marketing change, develops. Variety selection in Florida often requires special regional consideration due to the wide range of climatic variations of the peninsula. Some currently used legume varieties for Florida, are presented in Table 1. 


\section{SEEDING AND PLANTING}

Planting dates and seeding information for legumes are given in Table 2.

\section{FERTILIZER AND LIME}

Broadcast all $\mathrm{P}_{2} \mathrm{O}_{5}$ and micronutrients. Band 25 to $50 \%$ of $\mathrm{N}$ and $\mathrm{K}_{2} \mathrm{O}$ at planting. Sidedress remaining $\mathrm{N}$ and $\mathrm{K}_{2} \mathrm{O}$ at pre-bloom stage. Sidedress $\mathrm{N}$ and $\mathrm{K}_{2} \mathrm{O}$ can be applied through center pivot irrigation system. Soil test results for mineral soils for legumes are given in Table 3.

\section{PLANT TISSUE ANALYSIS}

Plant tissue analysis information for legumes is given in Table 4 . The analysis was done at early bloom, using the most recently matured trifoliate leaf.

\section{IRRIGATION}

Irrigation is critical when rainfall is low during the fruit set and pod development period. Crop water requirements (see Chapter 8, Principles and Practices of Irrigation Management for Vegetables, Tables 4-6) may approach $95 \%$ of ETo (see Chapter 8, , Table 3) during rapid growth and development. Thus, if ETo were around 0.15 inches per day, crop water use might average 0.14 inches per day (this equals about 3800 gallons per acre per day). If an overhead irrigation system were used and operated to apply water with a $70 \%$ efficiency, then the daily irrigation requirement would average 0.20 inches (5400 gallons per acre) [0.14/0.70]. Crop water requirements are expected to decrease to around $85 \%$ of ETo during the last period of crop growth.

Table 1. Legume varieties that are in commercial use in Florida arranged by type.

\begin{tabular}{|c|c|c|c|c|c|}
\hline Type & Variety & Type & Variety & Type & Variety \\
\hline $\begin{array}{l}\text { Green Bush } \\
\text { (Fig. 29-1) }\end{array}$ & $\begin{array}{l}\text { Ambra } \\
\text { Benchmark } \\
\text { Bronco } \\
\text { Capricorn } \\
\text { Charon } \\
\text { Dusky } \\
\text { Fandango } \\
\text { Hialeah } \\
\text { Leon } \\
\text { Mercury } \\
\text { Mirada } \\
\text { Opus }\end{array}$ & $\begin{array}{l}\text { Green Bush } \\
\text { Yellow Bush } \\
\text { Green Pole } \\
\text { (Fig. 29-2) } \\
\text { Lima }\end{array}$ & $\begin{array}{l}\text { Benchmark } \\
\text { Prosperity } \\
\text { Seville } \\
\text { Sonata } \\
\text { Storm } \\
\text { Gold Mine } \\
\text { Golden Rod } \\
\text { Gold Rush } \\
\text { Dade } \\
\text { Macaslan } \\
\text { Fordhook } 242\end{array}$ & $\begin{array}{l}\text { Lima } \\
\text { Southernpea }\end{array}$ & $\begin{array}{l}\text { Early Thorogreen } \\
\text { Jackson Wonder } \\
\text { Nemagreen } \\
\text { Knuckle Purplehull } \\
\text { Magnolia } \\
\text { Pinkeye Purplehull } \\
\text { Texas Cream } 40 \\
\text { White Acre } \\
\text { Zipper Cream } \\
\text { Oregon Sugarpod II }\end{array}$ \\
\hline
\end{tabular}

Table 2. Seeding and planting information for legumes in Florida.

\begin{tabular}{|c|c|c|c|c|c|c|}
\hline Planting dates & Snapbean bush & Snapbean pole & Lima bean bush & Lima bean pole & Southernpea & Snowpea \\
\hline North Florida & $\begin{array}{l}\text { Mar - Apr; } \\
\text { Aug - Sept }\end{array}$ & $\begin{array}{l}\text { Mar - Apr; } \\
\text { Aug - Sept }\end{array}$ & $\begin{array}{l}\text { Mar - Apr; } \\
\text { Aug - Sept }\end{array}$ & $\begin{array}{l}\text { Mar - Apr; } \\
\text { Aug - Sept }\end{array}$ & Mar - Jul & Jan - Mar \\
\hline Central Florida & $\begin{array}{l}\text { Feb - Apr; } \\
\text { Aug - Sept }\end{array}$ & $\begin{array}{l}\text { Feb - Apr; } \\
\text { Aug - Sept }\end{array}$ & $\begin{array}{l}\text { Feb - Mar; } \\
\text { Aug - Sept }\end{array}$ & $\begin{array}{l}\text { Feb - Mar; } \\
\text { Aug - Sept }\end{array}$ & Feb - Aug & Nov - Feb \\
\hline South Florida & Sept - Apr & Sept - Apr & Sept - Apr & Sept - Apr & Sept - Apr & Nov - Feb \\
\hline \multicolumn{7}{|c|}{ Seeding information } \\
\hline $\begin{array}{l}\text { Distance between } \\
\text { rows (in) }\end{array}$ & $18-36$ & $36-48^{1}$ & $18-36$ & $36-48^{1}$ & $20-42$ & $\begin{array}{l}36^{1} \\
2 \text { row bed }\end{array}$ \\
\hline $\begin{array}{l}\text { Distance between } \\
\text { plants (in) }\end{array}$ & $2-4$ & $3-5$ & $3-6$ & $8-12$ & $3-6$ & $2-6$ \\
\hline $\begin{array}{l}\text { Seeding depth (in) } \\
\text { Seed per acre (lb) }\end{array}$ & $\begin{array}{l}1-1.5 \\
45-80\end{array}$ & $\begin{array}{l}1-1.5 \\
30-45\end{array}$ & $\begin{array}{l}1-1.5 \\
40-60\end{array}$ & $\begin{array}{l}1-1.5 \\
20-40\end{array}$ & $\begin{array}{l}1-1.5 \\
15-30\end{array}$ & $\begin{array}{l}1-1.5 \\
25-50\end{array}$ \\
\hline $\begin{array}{l}\text { Days to maturity } \\
\text { from seed }\end{array}$ & $45-60$ & $50-70$ & $60-80$ & $80-100$ & $75-90$ & $60-80$ \\
\hline Plant populations ${ }^{2}$ & 172,240 & 58,000 & 116,160 & 21,780 & 104,544 & 87,120 \\
\hline
\end{tabular}




\section{WEED MANAGEMENT}

Herbicides labeled for beans are listed in Table 5.

\section{DISEASE MANAGEMENT}

Chemicals approved for disease management in legumes are outlines in tables as follows:

Table 6 - Bean

Table 7 - Southernpea

\section{INSECT MANAGEMENT}

Table 8 outlines the insecticides approved for use on insects attacking bean.

\section{PRODUCTION COSTS}

An example of breakeven production costs for snap bean is given in Table 9 .

Table 3. Soil test results and fertilizer recommendations for mineral soils for legumes. ${ }^{1}$

\begin{tabular}{|c|c|c|c|c|c|c|c|c|c|c|c|}
\hline \multirow[t]{2}{*}{ Target pH } & \multirow[t]{2}{*}{$\mathrm{N}$ Ib/A } & VL & $\mathrm{L}$ & M & $\mathrm{H}$ & VH & VL & $\mathbf{L}$ & M & $\mathrm{H}$ & VH \\
\hline & & \multicolumn{4}{|c|}{$\mathrm{P}_{2} \mathrm{O}_{5}{ }^{2}$} & $(\mathrm{Ib} / \mathrm{A} / \mathrm{c}$ & \multicolumn{5}{|c|}{$\mathrm{K}_{2} \mathrm{O}$} \\
\hline $\begin{array}{l}\text { Snapbean } \\
6.5 \\
\text { Southernpea }\end{array}$ & & 120 & 100 & 80 & 0 & 0 & 120 & 100 & 80 & 0 & 0 \\
\hline \multirow{2}{*}{\multicolumn{12}{|c|}{$\begin{array}{ll}\text { Southernpea, Snowpea, English pea } \\
6.5 & 60 \\
\text { Lima bean, Pole bean } & 80 \\
65 & 100\end{array}$}} \\
\hline 6.5 & & & & & & & & & & & \\
\hline
\end{tabular}

Table 4. Plant tissue analysis at early bloom for snapbean.

\begin{tabular}{|c|c|c|c|c|c|c|c|c|c|c|c|c|}
\hline \multirow[b]{2}{*}{ Status } & $\mathbf{N}$ & $\mathbf{P}$ & K & $\mathrm{Ca}$ & $\mathrm{Mg}$ & $S$ & $\mathrm{Fe}$ & Mn & $\mathrm{Zn}$ & B & $\mathrm{Cu}$ & Mo \\
\hline & \multicolumn{6}{|c|}{ Percent } & \multicolumn{6}{|c|}{ Parts per million } \\
\hline Deficient & $<3.0$ & 0.25 & 2.0 & 0.8 & 0.25 & 0.20 & 25 & 20 & 20 & 15 & 5 & 0.4 \\
\hline Adequate range & $3.0-4.0$ & $0.25-0.45$ & $2.0-3.0$ & $0.8-1.5$ & $0.25-0.45$ & $0.20-0.40$ & $25-200$ & $20-100$ & $20-40$ & $15-40$ & $5-10$ & $0.4-0.8$ \\
\hline High & $>4.0$ & 0.45 & 3.0 & 1.5 & 0.45 & 0.40 & 200 & 100 & 40 & 40 & 10 & 0.8 \\
\hline Toxic & & & & & & & & $>100$ & & $>150$ & & \\
\hline
\end{tabular}


Table 5. Chemical weed controls: Beans and Peas

\begin{tabular}{|c|c|c|c|c|}
\hline \multirow[b]{2}{*}{ Herbicide } & \multirow[b]{2}{*}{ Labeled crops } & \multirow{2}{*}{$\begin{array}{l}\text { Time of } \\
\text { application to crop }\end{array}$} & \multicolumn{2}{|c|}{ Rate (Ibs. Al./Acre) } \\
\hline & & & Mineral & Muck \\
\hline Bentazon (Basagran) & Beans, Peas & Early postemergence & $0.5-1.0$ & --- \\
\hline \multicolumn{5}{|c|}{$\begin{array}{l}\text { Remarks: Apply early postemergence when weeds are small and actively growing. Beans are to Basagran after the first trifoliate leaf ha } \\
\text { fully expanded. A crop oil concentrate or a UAN solution }(28,30,32 \% \text { nitrogen solution) may be added for improved control. Yellowing } \\
\text { bronzing, speckling or leaf burning may occur under certain conditions. This injury is generally outgrown without delaying podset or } \\
\text { maturity. Basagran is a contact herbicide and controls many young broadleaf weeds. It does not control grass. Read and follow the labe } \\
\text { directions for hard to control weeds such as yellow nutsedge. }\end{array}$} \\
\hline $\begin{array}{l}\text { Carfentrazone } \\
\text { (Aim) }\end{array}$ & $\begin{array}{l}\text { Legume Group } \\
\text { (All) }\end{array}$ & $\begin{array}{l}\text { Preplant } \\
\text { Directed-hooded } \\
\text { Row-middles }\end{array}$ & 0.031 & 0.031 \\
\hline
\end{tabular}

Remarks: Aim may be applied as a preplant burndown treatment and/or as a post-directed hooded application to row middles for the burndown of emerged broadleaf weeds. May be tank mixed with other registered herbicides. May be applied at up to $2 \mathrm{oz}(0.031 \mathrm{lb}$ ai). Use a quality spray adjuvant such as crop oil concentrate (coc) or non-ionic surfactant at recommended rates.

\begin{tabular}{llll}
\hline EPTC & Beans & Preplant incorporate or & $3.0-4.0$ \\
(Eptam 10G) & (Green or Dry) & at layby
\end{tabular}

(Eptam 7E)

Remarks: Controls germinating annuals and suppresses nutsedge and other perennial weeds. Incorporate in same operation to reduce evaporation loss. Direct layby applications between rows and incorporate.

\begin{tabular}{llll}
\hline \hline Glyphosate & Beans \& Peas & Chemical fallow & $0.3-1.0$ \\
(Roundup, Durango) & & Preplant, pre emergence, & \\
Touchdown, Glyphomax) & & Pre transplant
\end{tabular}

Remarks: Roundup, Glyphomax and Touchdown have several formulations. Check the label of each for specific labeling directions.

\begin{tabular}{|c|c|c|c|}
\hline $\begin{array}{l}\text { Halosulfuron } \\
\text { (Sandea) }\end{array}$ & Snap Beans & $\begin{array}{l}\text { Preemergence } \\
\text { Postemergence0.024-0.032 }\end{array}$ & $\begin{array}{c}0.024-0.032 \\
0.024-0.032\end{array}$ \\
\hline
\end{tabular}

Remarks: Apply preemergence application after planting but before cracking at $1 / 2$ to $2 / 3$ oz product/A. Use the lower reate on light sandy soils. Do not incorporate. For postemergence applications apply 1/2 to $2 / 3$ oz product 3 weekds after emergence or at the 3 trifoliate stage, but before flowering. Do not exceed 1 oz per acre per crop cycle. Apply with a non-ionic surfactant or crop oil concentrate.

\begin{tabular}{|c|c|c|c|}
\hline $\begin{array}{l}\text { Imazethapyr } \\
\text { (Pursuit) }\end{array}$ & $\begin{array}{l}\text { Dry Beans, Lima Beans, } \\
\text { Southern Peas, English Peas }\end{array}$ & $\begin{array}{l}\text { Preplant incorporated; } \\
\text { Preemergence; } \\
\text { Early Postemergence }\end{array}$ & $0.031-0.062$ \\
\hline
\end{tabular}

Remarks: May be applied to Navy, Great Northern, Red Kidney, Black turtle, Cranberry and small white type dry beans, Lima Beans, Southern and English Peas. May be applied preplant incorporated or Preemergence to all the above crops at 2 ozs/acre or 3 to 4 ozs/acre for English and Southern Peas. An early postmergence application at 3 ozs. (English Peas) and 4 ozs./acre (Southern Peas) may be made with a non-ionic surfactant. Controls a large number of broadleaf weeds preemergence and several postemergence. Read the label for weed species and timing for control.

\begin{tabular}{|c|c|c|c|c|}
\hline $\begin{array}{l}\text { Imazethapyr } \\
\text { (Pursuit) }\end{array}$ & Snap beans & $\begin{array}{l}\text { Preplant incorporated } \\
\text { Preemergence }\end{array}$ & 0.023 & 0.023 \\
\hline
\end{tabular}

Remarks: May be applied as a preplant incorporated or preemergence treatment to snapbeans at 1.5 oz. product/acre. May be tank-mixed with a registered preemergent grass herbicide. There is a 30 day PHI. Check plant back restrictions on the label.
S-Metolachlor
(Dual Magnum)
Pod Crops: Bush, Pole, Lima, Preplant incorporate or
Mung Beans; Southern, English Peas preemergence
$0.95-1.26 \quad 1.26$

Remarks: Dual Magnum is an isomer of Dual and has a lower application rate. Use 1 to 1.33 pints/A. Controls most annual grasses and some broadleaf weeds as well as yellow nutsedge. May be applied preplant incorporated or preemergence and watered into the soil. See label for specific tank-mix combinations and recommendations for Eptam and Treflan.

\begin{tabular}{|c|c|c|c|c|}
\hline $\begin{array}{l}\text { Paraquat } \\
\text { (Gramoxane Intron) } \\
\text { (Firestorm) }\end{array}$ & Lima, Snap Beans; Peas & Preplant Preemergence & $\begin{array}{l}0.47-0.94 \\
0.31-0.47\end{array}$ & $\begin{array}{l}0.47-0.94 \\
0.5-1.0\end{array}$ \\
\hline
\end{tabular}

Remarks: Apply as a band treatment over the crop row or as a broadcast treatment before, during or after planting, but before the emergence of the crop. Weeds emerging after the application will not be controlled. Crop plants emerged at the time of application will be killed. Use a non-ionic surfactant in the spray mixture. 
Table 5. Continued.

\begin{tabular}{|c|c|c|c|c|}
\hline \multirow[b]{2}{*}{ Herbicide } & \multirow[b]{2}{*}{ Labeled crops } & \multirow{2}{*}{$\begin{array}{l}\text { Time of } \\
\text { application to crop }\end{array}$} & \multicolumn{2}{|c|}{ Rate (Ibs. Al./Acre) } \\
\hline & & & Mineral & Muck \\
\hline $\begin{array}{l}\text { Paraquat } \\
\text { (Gramoxone Intron) } \\
\text { (Firestorm) }\end{array}$ & Dry beans & Harvest aid & $0.31-0.47$ & $0.31-0.47$ \\
\hline \multicolumn{5}{|c|}{$\begin{array}{l}\text { Remarks: Use a non-ionic spreader at } 1 \text { gt. per } 100 \text { gals. of spray mix. May be used in up to } 2 \text { applications. A split application may } \\
\text { improve vine coverage. Do not harvest within } 7 \text { days of last application. May be used on the dry forms of the following: Chick peas, } \\
\text { Garbanzo beans; Sweet, White sweet, White and Grain lupines; Kidney, Lima, Mung, Navy, Pinto, Snap and Wax beans; Asparagus beans } \\
\text { Blackeye and Cowpeas. Do not use on Faba beans. }\end{array}$} \\
\hline Pedimethalin (Prowl) & $\begin{array}{l}\text { Beans: Dry, Lima, Snap; Chickpeas, } \\
\text { Southern Peas }\end{array}$ & Preplant incorporated & $0.5-0.75$ & 1.0 \\
\hline \multicolumn{5}{|c|}{$\begin{array}{l}\text { Remarks: Incorporate within } 7 \text { days of application to the top } 1 \text { to } 2 \text { inches of soil. Label state control of many weeds including crab- } \\
\text { grass, fall and Texas panicum, goosegrass, signalgrass, carpetweed, Florida pusley, kochia, lambsquarter, pigweed, purslane and annual } \\
\text { spurges. May be applied alone or tank-mixed with Dual or Eptam. }\end{array}$} \\
\hline $\begin{array}{l}\text { Pelargonic Acid } \\
\text { (Scythe) }\end{array}$ & $\begin{array}{l}\text { Legume vegetables } \\
\text { (Beans (all) Peas (all)) }\end{array}$ & $\begin{array}{l}\text { Vegetative Burndown } \\
\text { (site preparation) }\end{array}$ & $3-10 \% \mathrm{v} / \mathrm{v}$ & $3-10 \% \mathrm{v} / \mathrm{v}$ \\
\hline
\end{tabular}

Remarks: General contact, non-selective, foliar applied herbicide. No residual control. Product is non-translocated. May be tank mixed with soil residual compounds. Consult label for rates and other information.

\begin{tabular}{|c|c|c|c|c|}
\hline $\begin{array}{l}\text { Quizalofop } \\
\text { (Assure II) } \\
\text { Targa }\end{array}$ & $\begin{array}{l}\text { Snap beans, dry beans, succulent } \\
\text { and dry peas }\end{array}$ & Postemergence & $0.04-0.08$ & $0.04-0.08$ \\
\hline
\end{tabular}

Remarks: For control of emerged annual and perennial grasses. Application is 6-12 oz Assure II to actively growing grasses depending on species to be controlled. Subsequent flushes of grasses require additional treatments. For ground application always include a nonphytotoxic petrolium based oil concentrate at $1 \% \mathrm{v} / \mathrm{v}(4 \mathrm{gts} / 100 \mathrm{gals})$ or a non ionic surfactant at $0.25 \% \mathrm{v} / \mathrm{v}$ ( $1 \mathrm{gt} / 100 \mathrm{gal})$. Reductions in grass control is possible when applied immediately prior to, or sequentially after application of post broadleaf herbicides. Follow label directions. It may be tank mixed with Basagran. Do not apply within 15 days of harvest. Do not apply more than 14 oz of product per acre per season.

\begin{tabular}{llll}
\hline Sethoxydim (Poast) & Beans and Peas, dry and succulent & Postemergence & 0.188 - 0.28
\end{tabular}

Remarks: For postemergence control of annual and perennial grass weeds, use 1 pt. (0.188 lb. ai.) to $1.5 \mathrm{pts}$. (0.28 lb. ai.) per acre depending on weed species to be controlled. Will not control sedges or broadleaf weeds. Use 2 pts. crop oil concentrate per acre in the spray mix. Do not apply more then 4 pts. per acre in one season. Do not apply within 15 days of harvest for succulent beans and peas or 30 days for dry beans and peas. Bean and pea types and species on which application may be made include beans of the Phaseolus genus (includes Adzuki bean, Field bean, Kidney bean, Lima bean, Navy bean, Mung bean); Lupines (includes Sweet lupine, White lupine, Grain lupine); Cowpeas - Vigna species (includes blackeye pea, Southern pea, Broad bean); Vicia faba or faba bean; Chick pea - Cicer arietinum or garbanzo bean; guar - Cyamopsis tetragoneloba; and peas - Pisum species (includes garden peas, field peas, sugar peas).

Sodium Chlorate (Defol 6) $\quad$ Dry beans; Southern peas; $\quad$ Defoliant/Desiccant 6.0
Guar beans

Remarks: Apply at a rate of 1 gal. per acre in 5 to 10 gals. of water by air or 10 to 20 gal. by ground equipment. Thorough coverage is essential. Make application 7 to 10 days before anticipated harvest, longer if temperatures are cool. Do not graze treated fields or feed treated fodder or forage to livestock.

\begin{tabular}{|lcc|}
\hline \hline Trifluralin & Green, Lima, Mung, Guar Beans; & Preplant incorporated \\
(Treflan EC) & Southern, English Peas & $0.5-0.75$ \\
(Treflan TR-10) & \\
(Treflan MTF) & \\
(Treflan 5) & \\
Remarks: Controls germinating annuals, especially grasses. Incorporate 4 inches or less within 8 hours. Results in Florida are erratic on \\
soils with low organic matter and clay contents. Note label precautions of planting non-registered crops. See labels for specific applica- \\
tion rates.
\end{tabular}


Table 6. Disease management for beans.

\begin{tabular}{|c|c|c|c|c|c|c|}
\hline Chemical & $\begin{array}{l}\text { Fungicide } \\
\text { Group1 }\end{array}$ & Maximum & $\begin{array}{l}\text { Rate/Acre/ } \\
\text { Season }\end{array}$ & $\begin{array}{l}\text { Min. Days } \\
\text { to Harvest }\end{array}$ & $\begin{array}{l}\text { Pertinent Diseases } \\
\text { or Pathogens }\end{array}$ & Remarks $^{2}$ \\
\hline \multicolumn{7}{|c|}{ Fungicides labeled on beans can be used on southern peas unless label restricts use for specific crops (e.g. snap beans, lima beans, etc) } \\
\hline $\begin{array}{l}\text { Amistar 80DF } \\
\text { (Azoxystrobin) }\end{array}$ & 11 & $50 z$ & $200 z$ & 0 & $\begin{array}{l}\text { Rust, web blight } \\
\text { White mold, } \\
\text { Anthracnose } \\
\text { Rhizoctonia diseases }\end{array}$ & $\begin{array}{l}\text { Do not exceed } 1 \text { sequential and } \\
4 \text { total applications of Amistar } \\
\text { or other Qol fungicides. }\end{array}$ \\
\hline $\begin{array}{l}\text { Apron XL LS } \\
\text { (Mefenoxam) }\end{array}$ & 4 & $\begin{array}{l}0.64 \mathrm{fl} .0 \mathrm{0z} . / \\
100 \mathrm{lb} \text { seed }\end{array}$ & & & Pythium seedling blight & Seed treatment only \\
\hline Basic Copper 53 & M1 & $4 \mathrm{lbs}$ & & 1 & Bacterial blights & \\
\hline $\begin{array}{l}\text { Basicop (Copper } \\
\text { sulfate) }\end{array}$ & & $4 \mathrm{lbs}$ & & 1 & Bacterial blights & \\
\hline Blocker 4F (PCNB) & 14 & $\begin{array}{l}3.3 \mathrm{oz} / 1000 \\
\text { row feet }\end{array}$ & & & White mold & At seeding \\
\hline \multirow{2}{*}{$\begin{array}{l}\text { Botran } 75 W \\
\text { (Dicloran) }\end{array}$} & 14 & 4 lbs (pole) & & 2 & Sclerotinia diseases & Snap beans only \\
\hline & & $\begin{array}{l}2.25 \mathrm{lbs} \\
\text { (bush) }\end{array}$ & & & & \\
\hline $\begin{array}{l}\text { Bravo Ultrex } \\
\text { (Chlorothalonil) }\end{array}$ & M5 & $2.7 \mathrm{lbs}$ & $7.3 \mathrm{lbs}$ & 7 & $\begin{array}{l}\text { Rust } \\
\text { Gray mold }\end{array}$ & \\
\hline Bravo Weather Stik, & M5 & $3 \mathrm{pts}$ & $12 \mathrm{pts}$ & 7 & Rust & \\
\hline $\begin{array}{l}\text { Echo 720, Equus } 720 \\
\text { SST (Chlorothalonil) }\end{array}$ & & & & & Gray mold & \\
\hline $\begin{array}{l}\text { Champ DP Dry Prill } \\
\text { (Copper hydroxide) }\end{array}$ & M1 & $2 \mathrm{lbs}$ & & 1 & Bacterial blights & \\
\hline Champ Formula $2 \mathrm{~F}$ & M1 & $2 \mathrm{pts}$ & & 1 & Bacterial blights & \\
\hline \multirow[t]{2}{*}{$\begin{array}{l}\text { Echo } 90 \text { DF, Equus- } \\
\text { DF (Chlorothalonil) }\end{array}$} & M5 & $\begin{array}{l}2.5 \mathrm{lbs} \\
\text { (snap) }\end{array}$ & & 7 & Rust & \\
\hline & & $\begin{array}{l}1.625 \mathrm{lbs} \\
\text { (dry) }\end{array}$ & & 14 & Gray mold & \\
\hline \multirow{2}{*}{$\begin{array}{l}\text { Endura 70WG } \\
\text { (Boscalid) }\end{array}$} & 7 & $11 \mathrm{oz}$ & $220 z$ & Dry 21 & Rust, Gray mold, & \\
\hline & & & & Succulent 7 & $\begin{array}{l}\text { White mold, Ascochyta } \\
\text { blight }\end{array}$ & \\
\hline $\begin{array}{l}\text { Fosphite (Potassium } \\
\text { Phosphite) }\end{array}$ & & $\begin{array}{l}1-3 \text { qt in } \\
\text { min of } 20 \\
\text { gal water }\end{array}$ & & & Various diseases & $\begin{array}{l}\text { Do not apply at less than } 3 \text { day } \\
\text { intervals }\end{array}$ \\
\hline $\begin{array}{l}\text { Headline } \\
\text { (Pyraclostrobin) }\end{array}$ & 11 & 6-9 fl. oz. & 18 fl. $0 \mathrm{z}$. & 21 & Various foliar diseases & $\begin{array}{l}\text { Do not exceed } 2 \text { applications in } \\
\text { one season }\end{array}$ \\
\hline $\begin{array}{l}\text { Kocide } 101 \text { (Copper } \\
\text { hydroxide) }\end{array}$ & M1 & $3 \mathrm{lbs}$ & & 1 & Bacterial blights & \\
\hline Kocide 2000 & M1 & $2.25 \mathrm{lbs}$ & & 1 & Bacterial blights & \\
\hline Kocide 4.5 LF & M1 & 2 pts & & 1 & Bacterial blights & \\
\hline Kocide DF & M1 & $3 \mathrm{lbs}$ & & 1 & Bacterial blights & \\
\hline Maneb 80WP & M3 & $2 \mathrm{lb}$ & $12 \mathrm{lb}$ & 30 & $\begin{array}{l}\text { Rust } \\
\text { Anthracnose }\end{array}$ & Dry beans only \\
\hline Maneb 75DF & M3 & $2 \mathrm{lb}$ & $12.8 \mathrm{lb}$ & 30 & $\begin{array}{l}\text { Rust } \\
\text { Anthracnose }\end{array}$ & Dry beans only \\
\hline Manex 4F (Maneb) & M3 & $1.6 \mathrm{qt}$ & $9.6 \mathrm{qt}$ & 30 & $\begin{array}{l}\text { Rust } \\
\text { Anthracnose }\end{array}$ & Dry beans only \\
\hline
\end{tabular}


Table 6. Continued.

\begin{tabular}{|c|c|c|c|c|c|c|}
\hline Chemical & $\begin{array}{l}\text { Fungicide } \\
\text { Group1 }\end{array}$ & Maximum & $\begin{array}{l}\text { Rate/Acre/ } \\
\text { Season }\end{array}$ & $\begin{array}{l}\text { Min. Days } \\
\text { to Harvest }\end{array}$ & $\begin{array}{l}\text { Pertinent Diseases } \\
\text { or Pathogens }\end{array}$ & Remarks $^{2}$ \\
\hline $\begin{array}{l}\text { Maxim 4FS } \\
\text { (Fludioxonil) }\end{array}$ & 12 & $\begin{array}{l}0.16 \mathrm{fl} \mathrm{oz/} \\
100 \mathrm{lbs} \text { of } \\
\text { seed }\end{array}$ & & & $\begin{array}{l}\text { Various seedling dis- } \\
\text { eases }\end{array}$ & Seed treatment only. \\
\hline $\begin{array}{l}\text { Nordox (Cuprous } \\
\text { oxide) }\end{array}$ & & $4 \mathrm{lb}$ & & & Bacterial blights & \\
\hline Nordox 75WG & & $2.5 \mathrm{lb}$ & & & Bacterial blights & \\
\hline $\begin{array}{l}\text { Nova } 40 \mathrm{~W} \\
\text { (Myclobutanil) }\end{array}$ & 3 & $50 z$ & $1.25 \mathrm{lbs}$ & 0 & $\begin{array}{l}\text { Rust } \\
\text { Rhizoctonia }\end{array}$ & Snap beans only \\
\hline $\begin{array}{l}\text { Nu-Cop 3L (Copper } \\
\text { hydroxide) }\end{array}$ & M1 & $4 \mathrm{pt}$ & & & Bacterial blights & \\
\hline Nu-Cop 50DF & M1 & $3 \mathrm{lb}$ & & & Bacterial blights & \\
\hline PCNB 2-E & & $1 \mathrm{gal}$ & & & $\begin{array}{l}\text { White mold } \\
\text { Foot rot }\end{array}$ & No applications after bloom \\
\hline $\begin{array}{l}\text { Quadris Flowable } \\
\text { (Azoxystrobin) }\end{array}$ & 11 & $15.4 \mathrm{fl} \mathrm{oz}$ & $2.88 \mathrm{qt}$ & 0 & $\begin{array}{l}\text { Rust, web blight } \\
\text { White mold, } \\
\text { Anthracnose } \\
\text { Rhizoctonia diseases }\end{array}$ & $\begin{array}{l}\text { Do not exceed } 1 \text { sequential and } \\
4 \text { total applications of Quadris } \\
\text { or other Qol fungicides. }\end{array}$ \\
\hline $\begin{array}{l}\text { Ridomil Gold EC } \\
\text { (Mefenoxam) }\end{array}$ & 4 & $1 \mathrm{pts}$ & & & $\begin{array}{l}\text { Pythium seedling dis- } \\
\text { eases }\end{array}$ & Apply at seeding \\
\hline $\begin{array}{l}\text { Ridomil Gold GR } \\
\text { (Mefenoxam; PCNB) }\end{array}$ & 4 and 14 & $\begin{array}{l}12 \mathrm{oz} / 1000 \\
\text { row feet }\end{array}$ & & & $\begin{array}{l}\text { Pythium seedling dis- } \\
\text { eases }\end{array}$ & Apply at seeding \\
\hline $\begin{array}{l}\text { Rovral 4F } \\
\text { Iprodione 4L }\end{array}$ & 2 & $2 \mathrm{pt}$ & $4 \mathrm{pt}$ & 14 & $\begin{array}{l}\text { Rhizoctonia } \\
\text { White mold }\end{array}$ & \\
\hline $\begin{array}{l}\text { Serenade Max } \\
\text { (Bacillus subtilis QST } \\
713 \text { ) }\end{array}$ & & $3 \mathrm{lb}$ & & 0 & $\begin{array}{l}\text { Rust } \\
\text { White mold }\end{array}$ & $\begin{array}{l}\text { For suppression or use as a } \\
\text { preventative in a program with } \\
\text { other registered fungicides. For } \\
\text { Sclerotinia, apply as a banded } \\
\text { spray (see label for placement } \\
\text { and timings). }\end{array}$ \\
\hline $\begin{array}{l}\text { Sonata (Bacillus } \\
\text { pumilus QST 2808) }\end{array}$ & & $4 \mathrm{qt}$ & & 0 & $\begin{array}{l}\text { Rust } \\
\text { White mold }\end{array}$ & $\begin{array}{l}\text { For suppression or use as a } \\
\text { preventative in a program with } \\
\text { other registered fungicides. }\end{array}$ \\
\hline $\begin{array}{l}\text { Sporan EC } \\
\text { (Rosemary oil; Clove } \\
\text { oil; Thyme oil) }\end{array}$ & & $\begin{array}{l}1-2 \text { pts. per } \\
\text { min of } 25 \\
\text { gal water }\end{array}$ & & & Various foliar diseases & \\
\hline $\begin{array}{l}\text { Stretch (Copper } \\
\text { hydroxide) }\end{array}$ & M1 & $6 \mathrm{pt}$ & & & Bacterial blights & \\
\hline $\begin{array}{l}\text { Sulfur (various } \\
\text { brands) }\end{array}$ & M2 & & & & & See label for rate \\
\hline $\begin{array}{l}\text { Switch (Cyprodinil; } \\
\text { Fludioxonil) }\end{array}$ & 9 and 12 & $11-14 \mathrm{oz}$. & $560 z$. & 7 & $\begin{array}{l}\text { White mold (Sclerotinia } \\
\text { sclerotiorum) Gray } \\
\text { Mold (Botrytis cinerea) }\end{array}$ & $\begin{array}{l}\text { After } 2 \text { applications alternate } \\
\text { with another fungicide with a } \\
\text { different mode of action for } 2 \\
\text { applications }\end{array}$ \\
\hline Terraclor 2E (PCNB) & 14 & 3 qts & & & Root and stem rot & Apply at planting \\
\hline Terraclor 75 WP & 14 & $2 \mathrm{lbs}$ & & & Root and stem rot & Apply at planting \\
\hline
\end{tabular}


Table 6. Continued.

\begin{tabular}{|c|c|c|c|c|c|c|}
\hline Chemical & $\begin{array}{l}\text { Fungicide } \\
\text { Group1 }\end{array}$ & $\frac{\text { Maximum } F}{\text { Application }}$ & $\frac{\text { Rate/Acre/ }}{\text { Season }}$ & $\begin{array}{l}\text { Min. Days } \\
\text { to Harvest }\end{array}$ & $\begin{array}{l}\text { Pertinent Diseases } \\
\text { or Pathogens }\end{array}$ & Remarks 2 \\
\hline Terraclor $\mathrm{F}$ & 14 & 3 pts & & & Root and stem rot & Apply at planting \\
\hline $\begin{array}{l}\text { Topaz (Potassium } \\
\text { Phosphite) }\end{array}$ & & $\begin{array}{l}1-3 q \mathrm{qt} / 100 \\
\text { gal water }\end{array}$ & $18 \mathrm{qt}$ & & Various diseases & $\begin{array}{l}\text { Do not exceed } 6 \text { applications } \\
\text { per a crop cycle or year }\end{array}$ \\
\hline \multirow[t]{3}{*}{$\begin{array}{l}\text { Topsin } 4.5 \mathrm{FL} \\
\text { (Thiophanate methyl) }\end{array}$} & 1 & $400 z$ & $800 z$ & $\begin{array}{l}14 \text { (snap } \\
\text { beans) }\end{array}$ & White mold & \\
\hline & & & & $\begin{array}{l}28 \text { (lima } \\
\text { beans) }\end{array}$ & Gray mold & \\
\hline & & & & & Anthracnose & \\
\hline \multirow[t]{3}{*}{ Topsin M 70 WP } & 1 & $2 \mathrm{lbs}$ & $4 \mathrm{lbs}$ & $\begin{array}{l}14 \text { (snap } \\
\text { beans) }\end{array}$ & White mold & \\
\hline & & & & $\begin{array}{l}28 \text { (lima } \\
\text { beans) }\end{array}$ & Gray mold & \\
\hline & & & & & Anthracnose & \\
\hline \multirow[t]{3}{*}{ Topsin M WSB } & 1 & $2 \mathrm{lbs}$ & $4 \mathrm{lbs}$ & $\begin{array}{l}14 \text { (snap } \\
\text { beans) }\end{array}$ & White mold & \\
\hline & & & & $\begin{array}{l}28 \text { (lima } \\
\text { beans) }\end{array}$ & Gray mold & \\
\hline & & & & & Anthracnose & \\
\hline Trilogy (Neem oil) & & $\begin{array}{l}0.5 \%-2.0 \% \\
\text { in } 25-100 \\
\text { gal of water }\end{array}$ & & & Various foliar diseases & $\begin{array}{l}\text { Do not exceed } 2 \text { gal Trilogy per } \\
\text { acre, per application }\end{array}$ \\
\hline \multicolumn{7}{|c|}{$\begin{array}{l}1 \text { Fungicide group (FRAC Code): Numbers (1-37) and letters (M, U, P) are used to distinguish the fungicide mode of action groups. All } \\
\text { fungicides within the same group (with same number or letter) indicate same active ingredient or similar mode of action. This informa- } \\
\text { tion must be considered for the fungicide resistance management decisions. } M=\text { Multi site inhibitors, fungicide resistance risk is low; } \\
U=\text { Recent molecules with unknown mode of action; } P=\text { host plant defense inducers. Source: http://www.frac.info/ (FRAC = Fungicide } \\
\text { Resistance Action Committee). Be sure to read a current product label before applying any chemicals. }\end{array}$} \\
\hline \multicolumn{7}{|c|}{$\begin{array}{l}2 \text { Information provided in this table applies only to Florida. Be sure to read a current product label before applying any chemical. The use } \\
\text { of brand names and any mention or listing of commercial products or services in the publication does not imply endorsement by the } \\
\text { University of Florida Cooperative Extension Service nor discrimination against similar products or services not mentioned. }\end{array}$} \\
\hline
\end{tabular}

Table 7. Disease management for southernpea.

\begin{tabular}{|c|c|c|c|c|c|}
\hline \multirow[b]{2}{*}{ Chemical } & \multicolumn{2}{|c|}{ Maximum Rate/Acre/ } & \multirow{2}{*}{$\begin{array}{l}\text { Minimum Days } \\
\text { to Harvest }\end{array}$} & \multirow[b]{2}{*}{ Pertinent Diseases } & \multirow[b]{2}{*}{ Select Remarks } \\
\hline & Application & Crop & & & \\
\hline \multicolumn{6}{|c|}{$\begin{array}{l}\text { Fungicides labelled on beans can be used on Southernpeas unless the label restricts use for specific crops (e.g. snap beans, lima beans, } \\
\text { etc). }\end{array}$} \\
\hline Ridomil Gold 4 EC & $1 \mathrm{pt} /$ trtd acre & & & $\begin{array}{l}\text { Pythium seedling } \\
\text { blights }\end{array}$ & $\begin{array}{l}\text { Apply at seeding in a 7-12" } \\
\text { band on soil over seed } \\
\text { furrow. }\end{array}$ \\
\hline Amistar 80 DF & 5 ozs & 20 ozs & 0 & $\begin{array}{l}\text { Rust, Cercospora } \\
\text { leaf spot }\end{array}$ & $\begin{array}{l}\text { Limit is } 1 \text { sequential appl. and } \\
4 \text { appl. per crop }\end{array}$ \\
\hline Bravo Ultrex 82.5 DF & $1.8 \mathrm{lbs}$ & $7.3 \mathrm{lbs}$ & 14 & $\begin{array}{l}\text { Rust, Cercospora } \\
\text { leaf spot }\end{array}$ & $\begin{array}{l}\text { Do not use crop } \\
\text { for livestock. }\end{array}$ \\
\hline Bravo Weather Stik 6 F & $2 \mathrm{pts}$ & $8 \mathrm{pts}$ & 14 & $\begin{array}{l}\text { Rust, Cercospora } \\
\text { leaf spot }\end{array}$ & $\begin{array}{l}\text { Limit is } 4 \text { appl./crop. } \\
\text { Not for use as animal feed. }\end{array}$ \\
\hline
\end{tabular}


Table 8. Selected insecticides approved for use on insects attacking beans and peas.

\begin{tabular}{|c|c|c|c|c|c|c|}
\hline $\begin{array}{l}\text { Trade Name } \\
\text { (Common Name) }\end{array}$ & $\begin{array}{l}\text { Rate } \\
\text { (product/acre) }\end{array}$ & $\begin{array}{l}\text { REl } \\
\text { (hours) }\end{array}$ & $\begin{array}{l}\text { Days to } \\
\text { Harvest }\end{array}$ & Insects & $\begin{array}{l}\text { MOA } \\
\text { Code } 1\end{array}$ & Notes \\
\hline $\begin{array}{l}\text { Admire } 2 \mathrm{~F} \\
\text { (imidacloprid) }\end{array}$ & $16-24$ oz & 12 & 21 & $\begin{array}{l}\text { aphids, leafhoppers, } \\
\text { thrips (foliar feeding), } \\
\text { whiteflies }\end{array}$ & $4 \mathrm{~A}$ & $\begin{array}{l}\text { Do not apply more than } 24 \mathrm{oz} \\
\text { product per acre per season. }\end{array}$ \\
\hline $\begin{array}{l}\text { Admire Pro } \\
\text { (imidacloprid) }\end{array}$ & $7-10.5 \mathrm{fl} \mathrm{oz}$ & 12 & 21 & $\begin{array}{l}\text { aphids, leafhoppers, } \\
\text { thrips, whiteflies }\end{array}$ & $4 \mathrm{~A}$ & $\begin{array}{l}\text { Do not apply more than } 10.5 \mathrm{fl} \\
\text { oz per acre per season. }\end{array}$ \\
\hline $\begin{array}{l}\text { Agree WG } \\
\text { (Bacillus thuringiensis } \\
\text { subspecies aizawai) }\end{array}$ & $0.5-2.0 \mathrm{lb}$ & 4 & 0 & $\begin{array}{l}\text { lepidopteran larvae (cat- } \\
\text { erpillar pests) }\end{array}$ & 11B1 & $\begin{array}{l}\text { Apply when larvae are small for } \\
\text { best control. OMRI-listed². }\end{array}$ \\
\hline $\begin{array}{l}\text { *Asana XL(0.66 EC) } \\
\text { (esfenvalerate) }\end{array}$ & $2.9-9.6 \mathrm{fl} \mathrm{oz}$ & 12 & $\begin{array}{l}3 \text { - snap } \\
21-\text { dry }\end{array}$ & $\begin{array}{l}\text { beet armyworm (aids } \\
\text { in control), cabbage } \\
\text { looper, corn earworm, } \\
\text { corn rootworm (adults), } \\
\text { cowpea curculio, cucum- } \\
\text { ber beetles, cutworms, } \\
\text { European corn borer, flea } \\
\text { beetles, grasshoppers, } \\
\text { green cloverworm, leaf- } \\
\text { hoppers, Mexican bean } \\
\text { beetle, painted lady but- } \\
\text { terfly (larvae), pea aphid, } \\
\text { saltmarsh caterpillar, } \\
\text { velvetbean caterpillar }\end{array}$ & 3 & $\begin{array}{l}\text { Do not feed or graze livestock on } \\
\text { treated vines. Do not apply more } \\
\text { than } 0.2 \mathrm{lb} \text { ai/acre per season ( } 4 \\
\text { applications at highest rate). }\end{array}$ \\
\hline $\begin{array}{l}\text { Aza-Direct } \\
\text { (azadirachtin) }\end{array}$ & $\begin{array}{l}1-2 \mathrm{pts} \\
(\max 3.5 \mathrm{pts})\end{array}$ & 4 & 0 & $\begin{array}{l}\text { aphids, beetles, cater- } \\
\text { pillars, leafhoppers, } \\
\text { leafminers, mites, stink } \\
\text { bugs, thrips, weevils, } \\
\text { whiteflies }\end{array}$ & 26 & $\begin{array}{l}\text { Antifeedant, repellent, insect } \\
\text { growth regulator. OMRI-listed }{ }^{2} \text {. }\end{array}$ \\
\hline $\begin{array}{l}\text { Azatin XL } \\
\text { (azadirachtin) }\end{array}$ & $5-21 \mathrm{fl} \mathrm{oz}$ & 4 & 0 & $\begin{array}{l}\text { aphids, beetles, cater- } \\
\text { pillars, leafhoppers, } \\
\text { leafminers, mites, stink } \\
\text { bugs, thrips, weevils, } \\
\text { whiteflies }\end{array}$ & 26 & $\begin{array}{l}\text { Antifeedant, repellent, insect } \\
\text { growth regulator. }\end{array}$ \\
\hline \multirow[t]{2}{*}{$\begin{array}{l}\text { *Baythroid } 2 \\
\text { (cyfluthrin) }\end{array}$} & $\begin{array}{l}0.8-3.2 \mathrm{fl} \mathrm{oz} \\
- \text { dry beans \& } \\
\text { peas }\end{array}$ & 12 & $\begin{array}{l}7 \text { - dry } \\
\text { beans \& } \\
\text { peas }\end{array}$ & 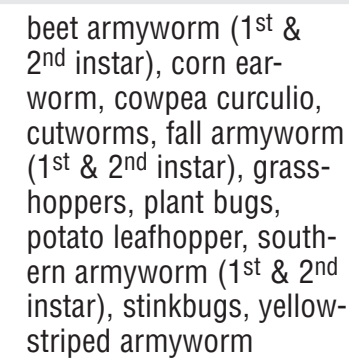 & 3 & $\begin{array}{l}\text { Maximum applications for dry } \\
\text { peas }=2 \text {. } \\
\text { Maximum for southern peas }=5 \text {. } \\
\text { Not for use on succulent beans } \\
\text { or peas or dry beans. }\end{array}$ \\
\hline & $\begin{array}{l}0.8-2.1 \mathrm{fl} \mathrm{oz}- \\
\text { southern pea }\end{array}$ & & $\begin{array}{l}3 \text { - } \\
\text { southern } \\
\text { pea }\end{array}$ & & & \\
\hline $\begin{array}{l}\text { Biobit HP } \\
\text { (Bacillus thuringiensis } \\
\text { subspecies kurstaki) }\end{array}$ & $0.5-2.0 \mathrm{lb}$ & 4 & 0 & $\begin{array}{l}\text { caterpillars (will not con- } \\
\text { trol large armyworms) }\end{array}$ & 11B2 & $\begin{array}{l}\text { Treat when larvae are young. } \\
\text { Good coverage is essential. } \\
\text { Can be used in the greenhouse. } \\
\text { OMRI-listed }{ }^{2} \text {. }\end{array}$ \\
\hline $\begin{array}{l}\text { BotaniGard } 22 \text { WP, ES } \\
\text { (Beauveria bassiana) }\end{array}$ & $\begin{array}{l}\text { WP: } \\
0.5-2 \mathrm{lb} / 100 \mathrm{gal} \\
\text { ES: } \\
0.5-2 \text { qts/100 } \\
\text { gal }\end{array}$ & 4 & 0 & aphids, thrips, whiteflies & -- & $\begin{array}{l}\text { May be used in greenhouses. } \\
\text { Contact dealer for recommen- } \\
\text { dations if an adjuvant must be } \\
\text { used. Not compatible in tank mix } \\
\text { with fungicides. }\end{array}$ \\
\hline
\end{tabular}


Table 8. Continued.

\begin{tabular}{|c|c|c|c|c|c|c|}
\hline $\begin{array}{l}\text { Trade Name } \\
\text { (Common Name) }\end{array}$ & $\begin{array}{l}\text { Rate } \\
\text { (product/acre) }\end{array}$ & $\begin{array}{l}\text { REI } \\
\text { (hours) }\end{array}$ & $\begin{array}{l}\text { Days to } \\
\text { Harvest }\end{array}$ & Insects & $\begin{array}{l}\text { MOA } \\
\text { Code } 1\end{array}$ & Notes \\
\hline $\begin{array}{l}\text { *Capture } 2 \text { EC } \\
\text { (bifenthrin) }\end{array}$ & $1.6-6.4 \mathrm{fl} \mathrm{oz}$ & 12 & 3 & $\begin{array}{l}\text { aphids, armyworms, bean } \\
\text { leaf beetle, cloverworm, } \\
\text { corn earworm, corn root- } \\
\text { worm adults, cucumber } \\
\text { beetles, loopers, Lygus } \\
\text { spp., mites, pea leaf } \\
\text { weevil, pea weevil, plant } \\
\text { bugs, sap beetles, stink } \\
\text { bugs, thrips, webworms, } \\
\text { whiteflies }\end{array}$ & 3 & $\begin{array}{l}\text { Do not apply more than } 12.8 \\
\text { ounces of product per acre per } \\
\text { season. Succulent beans and } \\
\text { peas only. }\end{array}$ \\
\hline $\begin{array}{l}\text { Courier } 70 \text { WP, 40SC } \\
\text { (buprofezin) }\end{array}$ & $\begin{array}{l}\text { 70WP: } 9 \text { oz } \\
\text { 40SC: } 9-13.6 \\
\text { fl oz. }\end{array}$ & 12 & 14 & whitefly nymphs & 16 & $\begin{array}{l}\text { For snap beans only. Allow } 14 \\
\text { days between applications. Do } \\
\text { not exceed } 0.76 \mathrm{lb} \text { ai/acre per } \\
\text { crop ( } 2 \text { applications at high rate) }\end{array}$ \\
\hline $\begin{array}{l}\text { Crymax WDG } \\
\text { (Bacillus thuringiensis } \\
\text { subspecies kurstaki) }\end{array}$ & $0.5-2 \mathrm{lb}$ & 4 & 0 & caterpillars & 11B2 & $\begin{array}{l}\text { Use high rate for armyworms. } \\
\text { Treat when larvae are young. }\end{array}$ \\
\hline $\begin{array}{l}\text { Deliver } \\
\text { (Bacillus thuringiensis } \\
\text { subspecies kurstaki) }\end{array}$ & $0.25-1.5 \mathrm{lb}$ & 4 & 0 & caterpillars & $11 \mathrm{~B} 2$ & $\begin{array}{l}\text { Use higher rates for armyworms. } \\
\text { OMRI-listed². }\end{array}$ \\
\hline $\begin{array}{l}\text { Dibrom 8E } \\
\text { (naled) }\end{array}$ & $1-1.5 \mathrm{pt}$ & 48 & 1 & $\begin{array}{l}\text { aphids, leafhoppers, } \\
\text { loopers, Lygus bugs, spi- } \\
\text { der mites }\end{array}$ & $1 \mathrm{~B}$ & Ground application only. \\
\hline $\begin{array}{l}\text { Dicofol 4E } \\
\text { (dicofol) }\end{array}$ & $1-3 \mathrm{pt}$ & 12 & $\begin{array}{l}21 \text { - dry, } \\
\text { green, } \\
\text { lima }\end{array}$ & twospotted mites & 20 & $\begin{array}{l}\text { No more than } 2 \text { applications per } \\
\text { season. }\end{array}$ \\
\hline $\begin{array}{l}\text { Dimethoate 4EC, } 2.67 \\
\text { (dimethoate) }\end{array}$ & $\begin{array}{l}\text { 4EC: } 0.5-1 \mathrm{pt} \\
2.67: 0.75-1.5 \\
\text { pt }\end{array}$ & 48 & $\begin{array}{l}2-4 E C \\
0-2.67\end{array}$ & $\begin{array}{l}\text { aphids, bean leaf beetle, } \\
\text { grasshoppers, leafhop- } \\
\text { pers, leafminers, Lygus } \\
\text { bug, Mexican bean beetle, } \\
\text { mites }\end{array}$ & 1B & $\begin{array}{l}\text { Do not feed treated vines. Highly } \\
\text { toxic to bees. }\end{array}$ \\
\hline $\begin{array}{l}\text { DiPel DF } \\
\text { (Bacillus thuringiensis } \\
\text { subspecies kurstaki) }\end{array}$ & $0.5-2 \mathrm{lb}$ & 4 & 0 & caterpillars & 11B2 & $\begin{array}{l}\text { Treat when larvae are young. } \\
\text { Good coverage is essential. } \\
\text { OMRI-listed?2. }\end{array}$ \\
\hline $\begin{array}{l}\text { *Di-Syston } 8 \mathrm{EC} ;{ }^{*} 15 \mathrm{G} \\
\text { (disulfoton) }\end{array}$ & $\begin{array}{l}\text { 8EC: } 1-2 \mathrm{pt} \\
\text { 15G: } 6.7 \mathrm{lb}\end{array}$ & 48 & $\begin{array}{l}\text { planting } \\
\text { time only } \\
\text { - green/ } \\
60 \text {-dry }\end{array}$ & $\begin{array}{l}\text { aphids, Mexican bean } \\
\text { beetle, mites, thrips }\end{array}$ & 1B & $\begin{array}{l}\text { See label for information on pos- } \\
\text { sible phytotoxicity problems. }\end{array}$ \\
\hline $\begin{array}{l}\text { Endosulfan } 3 \text { EC } \\
\text { (endosulfan) }\end{array}$ & $0.66-1.33$ qts & 24 & 3 & $\begin{array}{l}\text { aphids, armyworms, } \\
\text { bean leaf skeletonizer, } \\
\text { cowpea curculio, cucum- } \\
\text { ber beetles, cutworms, } \\
\text { flea beetles, leafhoppers, } \\
\text { Mexican bean beetle, } \\
\text { stink bugs, whiteflies }\end{array}$ & 2 & $\begin{array}{l}\text { Do not use on lima beans. Do } \\
\text { not make more than } 3 \text { applica- } \\
\text { tions per year. }\end{array}$ \\
\hline $\begin{array}{l}\text { Entrust } \\
\text { (spinosad) }\end{array}$ & $1-20 z$ & 4 & $\begin{array}{l}3 \\
28-d r y\end{array}$ & $\begin{array}{l}\text { armyworms, corn ear- } \\
\text { worm, leafminers, loop- } \\
\text { ers, thrips }\end{array}$ & 5 & $\begin{array}{l}\text { Succulent - Do not apply more } \\
\text { than } 9 \text { oz/acre per crop. } \\
\text { Dry - Do not apply more than } \\
3.75 \text { oz/acre per crop. OMRI- } \\
\text { listed2. }\end{array}$ \\
\hline $\begin{array}{l}\text { Esteem Ant Bait } \\
\text { ((pyriproxyfen) }\end{array}$ & $1.5-2.0 \mathrm{lb}$ & 12 & 1 & red imported fire ant & & \\
\hline
\end{tabular}


Table 8. Continued.

\begin{tabular}{|c|c|c|c|c|c|c|}
\hline $\begin{array}{l}\text { Trade Name } \\
\text { (Common Name) }\end{array}$ & $\begin{array}{l}\text { Rate } \\
\text { (product/acre) }\end{array}$ & $\begin{array}{l}\text { REI } \\
\text { (hours) }\end{array}$ & $\begin{array}{l}\text { Days to } \\
\text { Harvest }\end{array}$ & Insects & $\begin{array}{l}\text { MOA } \\
\text { Code } 1\end{array}$ & Notes \\
\hline $\begin{array}{l}\text { Extinguish } \\
((\mathrm{S}) \text {-methoprene) }\end{array}$ & $1-1.5 \mathrm{lb}$ & 4 & 0 & fire ants & $7 \mathrm{~A}$ & $\begin{array}{l}\text { Slow-acting IGR (insect growth } \\
\text { regulator). Best applied early } \\
\text { spring and fall where crop will be } \\
\text { grown. Colonies will be reduced } \\
\text { after three weeks and eliminated } \\
\text { after } 8 \text { to } 10 \text { weeks. May be } \\
\text { applied by ground equipment or } \\
\text { aerially. }\end{array}$ \\
\hline $\begin{array}{l}\text { Intrepid 2F } \\
\text { (methoxyfenozide) }\end{array}$ & $4-16 \mathrm{fl} \mathrm{oz}$ & 4 & 7 & $\begin{array}{l}\text { armyworms, corn ear- } \\
\text { worm (suppression), } \\
\text { loopers }\end{array}$ & 18 & $\begin{array}{l}\text { Do not apply more than } 64 \mathrm{fl} \\
\text { oz per acre per season or make } \\
\text { more than } 4 \text { applications per } \\
\text { season. }\end{array}$ \\
\hline $\begin{array}{l}\text { Javelin WG } \\
\text { (Bacillus thuringiensis } \\
\text { subspecies kurstaki) }\end{array}$ & $0.12-1.50 \mathrm{lbs}$ & 4 & 0 & $\begin{array}{l}\text { most caterpillars, but } \\
\text { not Spodoptera species } \\
\text { (armyworms). }\end{array}$ & $11 \mathrm{~B} 2$ & $\begin{array}{l}\text { Treat when larvae are young. } \\
\text { Thorough coverage is essential. } \\
\text { OMRI-listed². }^{\text {. }}\end{array}$ \\
\hline $\begin{array}{l}\text { Kelthane MF } 4 \\
\text { (dicofol) }\end{array}$ & $1-3 \mathrm{pt}$ & 12 & 21 & mites & 20 & $\begin{array}{l}\text { No more than } 2 \text { applications per } \\
\text { season. }\end{array}$ \\
\hline $\begin{array}{l}\text { Knack IGR } \\
\text { (pyriproxyfen) }\end{array}$ & $8-10 \mathrm{fl} \mathrm{oz}$ & 12 & 7 & $\begin{array}{l}\text { silverleaf whitefly, sweet } \\
\text { potato whitefly }\end{array}$ & $7 \mathrm{D}$ & $\begin{array}{l}\text { Do not make more than } 2 \text { appli- } \\
\text { cations per season. }\end{array}$ \\
\hline $\begin{array}{l}\text { *Lannate LV, *SP } \\
\text { (methomyl) }\end{array}$ & $\begin{array}{l}\text { LV: } \\
0.75-3 \mathrm{pts} \\
\text { SP: } \\
0.25-1.0 \mathrm{lb}\end{array}$ & 48 & $\begin{array}{l}\text { See } \\
\text { label: } \\
\text { varies } \\
\text { with } \\
\text { rate and } \\
\text { crop use }\end{array}$ & $\begin{array}{l}\text { aphids, beet armyworm, } \\
\text { corn earworm, cucumber } \\
\text { beetles, European corn } \\
\text { borer, fall armyworms, } \\
\text { leafhoppers, loopers, } \\
\text { Lygus bugs, Mexican } \\
\text { bean beetle, saltmarsh } \\
\text { caterpillar, thrips, varie- } \\
\text { gated cutworm, yellow- } \\
\text { striped armyworm }\end{array}$ & $1 \mathrm{~A}$ & \\
\hline $\begin{array}{l}\text { Lepinox WDG } \\
\text { (Bacillus thuringiensis } \\
\text { subspecies kurstaki) }\end{array}$ & $1.0-2.0 \mathrm{lb}$ & 12 & 0 & $\begin{array}{l}\text { for most caterpillars, } \\
\text { including beet armyworm } \\
\text { (see label) }\end{array}$ & $11 \mathrm{~B} 2$ & $\begin{array}{l}\text { Treat when larvae are small. } \\
\text { Thorough coverage is essential. }\end{array}$ \\
\hline $\begin{array}{l}\text { Malathion 8F } \\
\text { (malathion) }\end{array}$ & $1.5 \mathrm{pt}$ & 12 & 1 & $\begin{array}{l}\text { aphids, cucumber bee- } \\
\text { tles, mites, Mexican bean } \\
\text { beetle, potato leafhopper }\end{array}$ & $1 \mathrm{~B}$ & $\begin{array}{l}\text { Field \& greenhouse. Do not graze } \\
\text { or feed forage to livestock. }\end{array}$ \\
\hline $\begin{array}{l}\text { *MSR Spray } \\
\text { Concentrate (oxy- } \\
\text { demeton-methyl) }\end{array}$ & $2 \mathrm{pt}$ & 48 & 21 & leafhoppers, mites & $1 \mathrm{~B}$ & Lima beans only. \\
\hline $\begin{array}{l}\text { Mocap *15G, EC } \\
\text { (ethoprop) }\end{array}$ & $\begin{array}{l}\text { See label for } \\
\text { rates }\end{array}$ & 48 & $\begin{array}{l}\text { at plant- } \\
\text { ing }\end{array}$ & symphylans & $1 \mathrm{~B}$ & $\begin{array}{l}\text { Snap and lima beans. Do not } \\
\text { allow granules to contact seed. }\end{array}$ \\
\hline $\begin{array}{l}\text { M-Pede } \mathbf{4 9 \%} \text { EC } \\
\text { Soap, insecticidal }\end{array}$ & $1-2 \%$ VN & 12 & 0 & $\begin{array}{l}\text { aphids, leafhoppers, } \\
\text { mites, plant bugs, thrips, } \\
\text { whiteflies }\end{array}$ & -- & OMRI-listed². \\
\hline $\begin{array}{l}\text { *Mustang Max } \\
\text { (zeta-cypermethrin) }\end{array}$ & $1.28-4.0 \mathrm{oz}$ & 12 & $\begin{array}{l}1 \text { - suc- } \\
\text { culent } \\
21 \\
- \text { dried } \\
\text { shelled } \\
\text { peas or } \\
\text { beans }\end{array}$ & $\begin{array}{l}\text { corn earworm, cowpea } \\
\text { curculio, cutworms, fall } \\
\text { armyworm, flea beetles, } \\
\text { grasshoppers, leafhop- } \\
\text { pers, lesser cornstalk } \\
\text { borer (aides in control), } \\
\text { Mexican bean beetle, } \\
\text { plant bugs, saltmarsh } \\
\text { caterpillar, southern } \\
\text { armyworm, stink bugs, } \\
\text { true armyworm, velvet- } \\
\text { bean caterpillar, yellow- } \\
\text { striped armyworm }\end{array}$ & 3 & $\begin{array}{l}\text { Do not make applications less } \\
\text { than } 5 \text { days apart. }\end{array}$ \\
\hline
\end{tabular}


Table 8. Continued.

\begin{tabular}{|c|c|c|c|c|c|c|}
\hline $\begin{array}{l}\text { Trade Name } \\
\text { (Common Name) }\end{array}$ & $\begin{array}{l}\text { Rate } \\
\text { (product/acre) }\end{array}$ & $\begin{array}{l}\text { REI } \\
\text { (hours) }\end{array}$ & $\begin{array}{l}\text { Days to } \\
\text { Harvest }\end{array}$ & Insects & $\begin{array}{l}\text { MOA } \\
\text { Code } 1\end{array}$ & Notes \\
\hline $\begin{array}{l}\text { Neemix } 4.5 \\
\text { (azadirachtin) }\end{array}$ & $4-16 \mathrm{fl} \mathrm{oz}$ & 12 & 0 & $\begin{array}{l}\text { aphids, armyworms, } \\
\text { bean leaf beetle, cabbage } \\
\text { looper, corn earworm, } \\
\text { cutworms, garden } \\
\text { webworm, leafminers, } \\
\text { loopers, soybean looper, } \\
\text { webworms, whiteflies }\end{array}$ & 26 & $\begin{array}{l}\text { Acts as IGR and feeding repel- } \\
\text { lent. Does not kill adult insects. } \\
\text { OMRI-listed². }\end{array}$ \\
\hline $\begin{array}{l}\text { Orthene 75S, } 97 \\
\text { (acephate) }\end{array}$ & $\begin{array}{l}\text { 75S: } 0.33-1.33 \\
\mathrm{lb} \\
\text { 97: } 0.25-1.0 \mathrm{lb}\end{array}$ & 24 & $\begin{array}{l}14 \\
\text { - snap } \\
\text { beans } \\
\text { or dry } \\
\text { beans } \\
\\
0 \text { - lima } \\
\text { beans, } \\
\text { suc- } \\
\text { culent } \\
\text { form }\end{array}$ & $\begin{array}{l}\text { aphids (excluding black } \\
\text { bean aphid), army- } \\
\text { worms (excluding beet } \\
\text { armyworm), bean leaf } \\
\text { beetle, bean leafroller, } \\
\text { cabbage looper, corn } \\
\text { earworm, cutworms, } \\
\text { European corn borer, } \\
\text { fleahoppers, grasshop- } \\
\text { pers, green cloverworm, } \\
\text { leafhoppers, Mexican } \\
\text { bean beetle, plant bugs } \\
\text { (Lygus), soybean looper, } \\
\text { thrips, whiteflies (except } \\
\text { silverleaf or sweetpotato } \\
\text { whiteflies) }\end{array}$ & $1 \mathrm{~B}$ & $\begin{array}{l}\text { Do not apply more than } 2 \mathrm{lb} \\
\text { active ingredient per acre per } \\
\text { season. }\end{array}$ \\
\hline $\begin{array}{l}\text { *Penncap-M } \\
\text { (methyl parathion) }\end{array}$ & $2-4 \mathrm{pts}$ & $\begin{array}{l}4 \text { days } \\
\text { (see } \\
\text { label) }\end{array}$ & 15 & $\begin{array}{l}\text { aphids, cowpea curcu- } \\
\text { lio, cucumber beetles, } \\
\text { European corn borer, } \\
\text { leafhoppers, Lygus bugs, } \\
\text { Mexican bean beetle, } \\
\text { stink bugs }\end{array}$ & $1 \mathrm{~B}$ & $\begin{array}{l}\text { For dry beans (southern peas) } \\
\text { Begin applications when blooms } \\
\text { are first observed. }\end{array}$ \\
\hline $\begin{array}{l}\text { *Proaxis Insecticide } \\
\text { (gamma-cyhalothrin) }\end{array}$ & $1.92-3.84 \mathrm{fl} \mathrm{oz}$ & 24 & $\begin{array}{l}7 \text { for } \\
\text { edible } \\
\text { podded } \\
\text { and suc- } \\
\text { culent } \\
\text { shelled. } \\
21 \text { for } \\
\text { dry } \\
\text { beans } \\
\text { and } \\
\text { peas. }\end{array}$ & $\begin{array}{l}\text { Aphids(1), armyworms(2), } \\
\text { bean leaf beetle, blister } \\
\text { beetles, corn earworm, } \\
\text { cucumber beetles, } \\
\text { cowpea curculio( }(3) \text {, } \\
\text { cutworms, flea beetles, } \\
\text { grasshoppers, green } \\
\text { cloverworm, leafhoppers, } \\
\text { leaftiers, lesser cornstalk } \\
\text { borer(1), loopers, mead- } \\
\text { ow spittlebug, Mexican } \\
\text { bean beetle, painted lady } \\
\text { butterfly (larvae), plant } \\
\text { bugs, saltmarsh caterpil- } \\
\text { lar, spider mites(1), stink } \\
\text { bugs, thrips }(1) \text {, tobacco } \\
\text { budworm, velvetleaf } \\
\text { caterpillar, webworms, } \\
\text { whiteflies (1) }\end{array}$ & 3 & $\begin{array}{l}\text { (1) Suppression only } \\
\text { (2) First and second instars only. } \\
\text { (3) For control before larvae bore } \\
\text { into the plant stalk or pods. } \\
\text { Do not apply more than } 1.92 \\
\text { pints per acre per season. }\end{array}$ \\
\hline $\begin{array}{l}\text { Provado } 1.6 \mathrm{~F} \\
\text { (imidacloprid) }\end{array}$ & $3.5 \mathrm{oz}$ & 12 & 7 & $\begin{array}{l}\text { aphids, leafhoppers, } \\
\text { whiteflies }\end{array}$ & $4 \mathrm{~A}$ & $\begin{array}{l}\text { Not recommended following a } \\
\text { soil application of Admire (suc- } \\
\text { culent \& edible podded only). }\end{array}$ \\
\hline $\begin{array}{l}\text { Pyrellin EC } \\
\text { (pyrethrins + rotenone) }\end{array}$ & $1-2 \mathrm{pt}$ & 12 & 12 hours & $\begin{array}{l}\text { aphids, bean leaf beetle, } \\
\text { cucumber beetles, } \\
\text { European corn borer, } \\
\text { flea beetles, fleahoppers, } \\
\text { leafhoppers, leafminers, } \\
\text { loopers, Lygus bugs, } \\
\text { mites, plant bugs, stink } \\
\text { bugs, thrips, whiteflies }\end{array}$ & 3,21 & \\
\hline
\end{tabular}


Table 8. Continued.

\begin{tabular}{|c|c|c|c|c|c|c|}
\hline $\begin{array}{l}\text { Trade Name } \\
\text { (Common Name) }\end{array}$ & $\begin{array}{l}\text { Rate } \\
\text { (product/acre) }\end{array}$ & $\begin{array}{l}\text { REI } \\
\text { (hours) }\end{array}$ & $\begin{array}{l}\text { Days to } \\
\text { Harvest }\end{array}$ & Insects & $\begin{array}{l}\text { MOA } \\
\text { Code } 1\end{array}$ & Notes \\
\hline $\begin{array}{l}\text { Sevin 80S, } 4 \mathrm{~F} \\
\text { (carbaryl) }\end{array}$ & $\begin{array}{l}\text { 80S: } 0.63-1.88 \\
\mathrm{lb} \\
\text { 4F: } 0.5-1.5 \mathrm{qt}\end{array}$ & 12 & $\begin{array}{l}14 \text { days } \\
\text { for graz- } \\
\text { ing or } \\
\text { harvest } \\
\text { for for- } \\
\text { age, or } \\
\text { within } 3 \\
\text { days of } \\
\text { harvest } \\
\text { of fresh } \\
\text { beans or } \\
\text { peas, or } \\
\text { within } 21 \\
\text { days of } \\
\text { harvest } \\
\text { of dried } \\
\text { beans } \\
\text { or peas, } \\
\text { seed or } \\
\text { hay. }\end{array}$ & $\begin{array}{l}\text { armyworms, bean leaf } \\
\text { beetle, blister beetles, } \\
\text { corn earworm, cowpea } \\
\text { curculio, cucumber } \\
\text { beetles, cutworms, fall } \\
\text { armyworm, flea beetles, } \\
\text { garden webworm, green } \\
\text { cloverworm, leafhoppers, } \\
\text { Mexican bean beetle, } \\
\text { plant bugs, stink bugs, } \\
\text { tarnished plant bug, } \\
\text { three-cornered alfalfa } \\
\text { hopper, thrips, velvetbean } \\
\text { caterpillar, webworms }\end{array}$ & $1 \mathrm{~A}$ & $\begin{array}{l}\text { Repeat, as needed, up to } 4 \text { times. } \\
\text { Applications should be at least } 7 \\
\text { days apart. }\end{array}$ \\
\hline $\begin{array}{l}\text { SpinTor } 2 \text { SC } \\
\text { (spinosad) }\end{array}$ & $3-6 \mathrm{fl} 0 \mathrm{z}$ & 4 & $\begin{array}{l}3 \text { - suc- } \\
\text { culent } \\
28 \text { - dry }\end{array}$ & $\begin{array}{l}\text { armyworms, corn ear- } \\
\text { worm, European corn } \\
\text { borer (eggs and larvae), } \\
\text { leafminers, loopers, } \\
\text { thrips }\end{array}$ & 5 & \\
\hline $\begin{array}{l}\text { Sun Spray } \mathbf{9 8 . 8 \%} \text {, } \\
\text { JMS Stylet-0il, others } \\
\text { (oil, insecticidal) }\end{array}$ & $\begin{array}{l}3-6 \mathrm{qts} / 100 \mathrm{gal} \\
\text { (JMS) } \\
1-2 \mathrm{gal} / 100 \mathrm{gal} \\
\text { (others) }\end{array}$ & 4 & 0 & $\begin{array}{l}\text { aphids, leafhoppers, } \\
\text { leafminers, mites, thrips, } \\
\text { whiteflies }\end{array}$ & -- & $\begin{array}{l}\text { Organic Stylet -Oil is } \\
\text { OMRI-listed². }^{2}\end{array}$ \\
\hline $\begin{array}{l}\text { *Temik 15G } \\
\text { (aldicarb) }\end{array}$ & $3.5-7.0 \mathrm{lb}$ & 48 & $\begin{array}{l}\text { at plant- } \\
\text { ing, } 90\end{array}$ & $\begin{array}{l}\text { aphids, leafhoppers, } \\
\text { leafminers, Mexican bean } \\
\text { beetle, mites }\end{array}$ & $1 \mathrm{~A}$ & $\begin{array}{l}\text { Dry beans only. One application. } \\
\text { Do not feed green forage hay, or } \\
\text { straw to livestock. Do not use } \\
\text { green pods as food for humans. }\end{array}$ \\
\hline $\begin{array}{l}{ }^{*} \text { Thimet } 20 \text { G } \\
\text { (phorate) }\end{array}$ & $\begin{array}{l}\text { No more than } \\
7.6 \mathrm{lb}\end{array}$ & 48 & 60 & $\begin{array}{l}\text { aphids, leafhoppers, } \\
\text { Lygus bugs, Mexican } \\
\text { bean beetles, mites, seed- } \\
\text { corn maggots, thrips }\end{array}$ & $1 \mathrm{~B}$ & $\begin{array}{l}\text { At planting only. Avoid direct } \\
\text { contact with seed. }\end{array}$ \\
\hline $\begin{array}{l}\text { Trigard } \\
\text { (cyromazine) }\end{array}$ & $2.66 \mathrm{oz}$ & 12 & 7 & leafminers & 17 & $\begin{array}{l}\text { Dry beans (including southern } \\
\text { pea), except cowpea, also suc- } \\
\text { culent lima beans. Limited to } 6 \\
\text { applications. }\end{array}$ \\
\hline $\begin{array}{l}\text { Trilogy } \\
\text { (extract of neem oil) }\end{array}$ & $0.5-2 \%$ V/V & 4 & 0 & $\begin{array}{l}\text { aphids, mites, suppres- } \\
\text { sion of thrips and white- } \\
\text { flies }\end{array}$ & 26 & $\begin{array}{l}\text { Apply morning or evening to } \\
\text { reduce potential for leaf burn. } \\
\text { Toxic to bees exposed to direct } \\
\text { treatment. OMRI-listed². }\end{array}$ \\
\hline
\end{tabular}


Table 8. Continued.

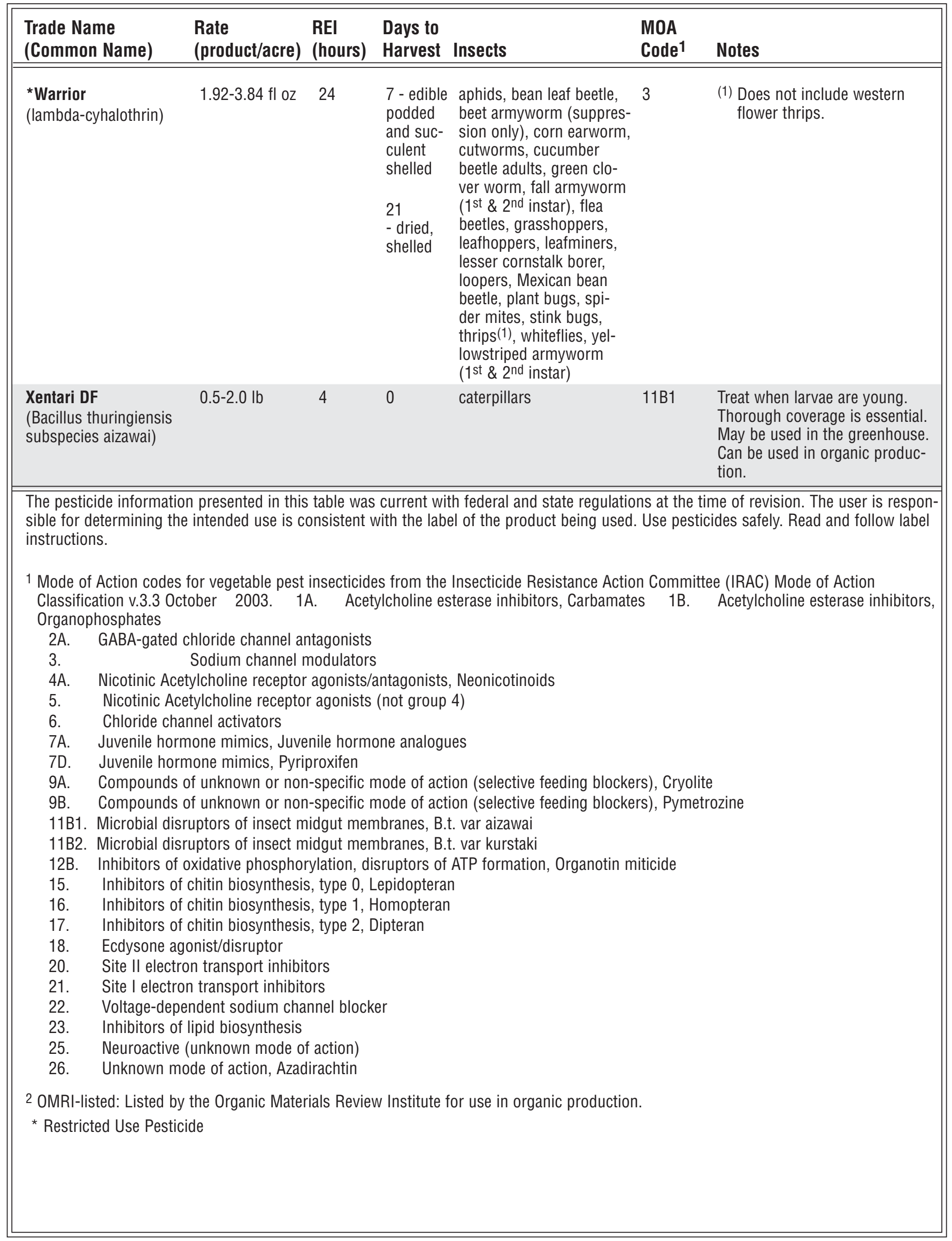


Table 9 . Breakeven production costs for snap bean at various yield levels in the Miami-Dade County area, 2004-2005.

\begin{tabular}{|c|c|c|c|c|c|c|}
\hline & \multirow[b]{2}{*}{ Cost per acre } & \multicolumn{5}{|c|}{ Yield (bushel/acre) } \\
\hline & & 185 & 210 & 235 & 260 & 285 \\
\hline Variable Costs & $\$ 1,553.10$ & $\$ 8.40$ & $\$ 7.40$ & $\$ 6.61$ & $\$ 5.97$ & $\$ 5.45$ \\
\hline Fixed Costs & $\$ 1,089.18$ & $\$ 5.89$ & $\$ 5.19$ & $\$ 4.63$ & $\$ 4.19$ & $\$ 3.82$ \\
\hline Harvest Cost/unit & & $\$ 6.40$ & $\$ 6.40$ & $\$ 6.40$ & $\$ 6.40$ & $\$ 6.40$ \\
\hline Total Cost/unit & & $\$ 20.68$ & $\$ 18.98$ & $\$ 17.64$ & $\$ 16.56$ & $\$ 15.67$ \\
\hline
\end{tabular}

